\title{
A Pilot Study Comparing Tools for Tracking Implementation Strategies and Treatment Adaptations
}

\section{Callie Walsh-Bailey}

Washington University in Saint Louis

Lorella G. Palazzo

Kaiser Permanente Washington Health Research Institute

Salene M. W. Jones

Fred Hutchinson Cancer Research Center

Kayne D. Mettert

Kaiser Permanente Washington Health Research Institute

\section{Byron J. Powell}

Washington University in Saint Louis

Shannon Wiltsey Stirman

Stanford University Department of Psychiatry and Behavioral Sciences

\section{Aaron R. Lyon}

University of Washington Department of Psychiatry and Behavioral Sciences

\section{Paul Rohde}

Oregon Research Institute

Cara C Lewis ( $\nabla$ cara.c.lewis@kp.org )

Kaiser Permanente Washington Health Research Institute https://orcid.org/0000-0001-8920-8075

\section{Methodology}

Keywords: implementation strategy, treatment adaptation, tailoring, tracking

Posted Date: January 13th, 2020

DOl: https://doi.org/10.21203/rs.2.20638/v1

License: (c) (i) This work is licensed under a Creative Commons Attribution 4.0 International License. Read Full License 


\section{Abstract}

\section{Background}

Tailoring implementation strategies and adapting treatments to better fit the local context may improve their effectiveness. However, there is a dearth of valid, reliable, pragmatic measures that allow for the prospective tracking of strategies and adaptations according to reporting recommendations. This study describes the development and pilot testing of three tools to designed to serve this purpose.

Methods

Measure development was informed by two systematic reviews of the literature (implementation strategies, treatment adaptation). The three resulting tools vary with respect to the degree of structure (Brainstorming Log $=$ low, Activity Log = moderate, Detailed Tracking Log $=$ high). To prospectively track treatment adaptations and implementation strategies, three stakeholder groups (treatment developer, implementation practitioners, mental health providers) were randomly assigned one tool per week via an anonymous web-based survey for nine weeks and incentivized to participate. Three established implementation outcome measures, the Acceptability of Intervention Measure, Intervention Appropriateness Measure, and Feasibility of Intervention Measure were used to assess the tools. Semistructured interviews were conducted to gather more nuanced information from stakeholders regarding their perceptions of the tools and the tracking process.

Results

The three tracking tools demonstrated moderate to good acceptability, appropriateness, and feasibility; the Activity Log was deemed the most feasible of the three tools. Implementation practitioners rated the tools the highest of the three stakeholder groups. The tools took an average of 15 minutes or less to complete.

\section{Conclusion}

This study sought to fill methodological gaps that prevent stakeholders and researchers from discerning which strategies are most important to deploy for promoting implementation and sustainment of evidence-based practices. These tools would allow researchers and practitioners to track whether activities were treatment adaptations or implementation strategies and what barrier(s) each targets. These tools could inform prospective tailoring of implementation strategies and treatment adaptations, which would promote scale out and spread.

\section{Background}

Mounting evidence suggests that tailoring implementation strategies to treatment context may be more effective than standardized approaches (1-4). Further, treatments may be optimized when they are carefully and prospectively adapted to better suit the contexts in which they are delivered $(5,6)$. Together, 
tailoring implementation strategies and adapting treatments may improve the sustainability of evidencebased practices (EBPs) (5). Tailoring and adapting frequently are reactive processes, occurring in an unplanned way throughout the course of an implementation effort (7); however, these activities may best achieve their desired effect through thoughtful and prospective planning (5). Both tailoring strategies and adapting treatment are highly complex processes that may benefit from tools to prompt and guide the consideration of implicated factors, but no such tools exist, to our knowledge.

Moreover, careful measurement of these tailoring and adapting processes is needed to evaluate their independent and interactive impact on targets and outcomes of interest. Currently, there are no valid, reliable, pragmatic tools that allow for planning or tracking the nuances of strategy tailoring and treatment adaptations. Two studies offer preliminary methods for tracking implementation strategies (8, 9). First, Bunger and colleagues (8) created an activity log in which participants in implementation efforts were asked to record implementation activities, their purpose, the actors involved, and time spent on the activity. This log was intended to collect both retrospective and prospective data in a low burden manner. This method offers an approach to capture information with sufficient detail to inform process evaluations or to replicate strategies. Data extracted allowed for identification of the action, actor, temporality, and dose of the implementation strategy. However, reporting according to recommendations (10) is limited as the justification, strategy target, and outcome are not explicit, rather had to be inferred by the research team. These early efforts confirm the importance of contemporaneous tracking as individuals involved in implementation can have difficulty remembering implementation events and activities when asked to recall these retrospectively (8).

Second, in the context of a randomized trial comparing standardized and tailored implementation approaches, Boyd et al. (9) developed a coding system to track implementation strategies and map them onto reporting recommendations offered by Proctor et al. (10). This coding system captured the same categories as Bunger et al.'s (8) activity log, as well as the strategy target, justification, and outcome. In theory, because of this alignment, the coding system generates sufficient detail to allow the implementation strategy to be reproduced, but this must be tested. Practical limitations imposed by coding, such as the substantive expertise needed, inability to track prospectively, time intensiveness, and limited feasibility outside the research context will need to be addressed through future work.

Although adapting treatment is considered one of $73+$ implementation strategies(11), a 2013 review of treatment adaptations (12) and a resulting framework illustrates a host of complexities that would not be captured by these two existing methods (13). This framework categorizes adaptations as those made to the treatment content (e.g. delivery and components of the EBP), context (e.g. setting or mode of delivery), or training and evaluation (e.g. "behind the scenes" implementation.) To understand how adaptations influence clinical and implementation outcomes, a feasible and reliable tracking tool that explicitly focuses on treatment adaptation is sorely needed. Without tracking, we are unable to discern if an adaptation constitutes drift or leads to improvements beyond what is expected of an EBP (14). 
Persistent knowledge and methodological gaps limit the degree to which researchers and practitioners can prospectively inform implementation tailoring and treatment adaptations. Without tools for prospective tracking, the field is hampered in its ability to disentangle which activity is most important, to what degree, and when for promoting sustainment. The perspectives of implementation practitioners, clinicians, and others who influence and are involved in implementation efforts are crucial to informing, evaluating, replicating, and scaling these efforts. Thus, any tool to support planning or tracking implementation strategies and treatment adaptation in real time ought to engage stakeholders; however, existing methods have largely been researcher driven.

The long-term objective of our work is to generate valid, reliable, and pragmatic tools that can be utilized in a variety of settings and treatment contexts by diverse end-users to prospectively guide and contemporaneously track tailoring of implementation strategies and treatment adaptations. The current manuscript reports on a mixed methods pilot test of three tools to prospectively track implementation strategies and treatment adaptations. The aims of the pilot study were to a) assess the utility, acceptability, feasibility, and appropriateness of each tool, b) assess the extent to which stakeholders adapted the EBP of interest and the nature of those adaptations, and c) gather preliminary evidence of the validity and reliability of the three tools. The results of this pilot work will inform subsequent refinement of the tools for optimal impact.

\section{Method}

\section{Tool Development}

The study Principal Investigator (PI; CCL) and the first author (CWB) reviewed available literature to locate examples of tracking tools regardless of substantive focus (e.g., journaling prompts, brainstorming activities, Bunger et al.'s activity log (8) and Boyd and colleagues' coding system (9)). We developed three tools in this pilot that varied on the degree to which they used open ended or structured/forced response options. There are tradeoffs to the degree of structure used (i.e., open versus forced), which may impact the completeness, quality, and actionability of the data collected. Greater reliability is associated with highly structured tools due to the standardization of response options (15). Less structured tools can allow for greater content validity and comprehensive qualitative coverage of a construct because participants are not limited in their responses, which can enhance content coverage beyond responses considered by the measure developer (16). However, in addition to allowing for a more diverse collection of responses, Reja el al. (17) note that open-ended questions require more extensive coding and are more prone to missing data (17). Edwards (15) indicated that open-ended questions may increase participant burden, while close-ended questions may be subject to bias, whether imposed by the investigator's lens or participant avoidance of selecting extreme options.

Despite the variability in the degree of structure, all three tools sought to capture the same categories of information (Table 1). The tools were informed and evaluated by three implementation frameworks and compilations (Supplemental file 1). One, Proctor and colleagues (10) offered detailed recommendations 
for implementation strategy reporting and specification to enable replication in research and practice (Figure 1). Tools varied in the degree to which responses aligned with these recommendations. Two, Lyon and colleagues $(18,19)$ created the SISTER compilation, in which existing implementation strategy labels and definitions were revised to improve fit with school settings, with several new strategies created and strategies not relevant to school settings removed. Only the most structured tool offered these standardized strategy labels, and in the case of the other two tools the SISTER compilation was applied to evaluate the quality of the responses. Three, Wiltsey Stirman and colleagues (13) put forth a system for classifying and reporting treatment adaptations that illuminates whether modifications made to an EBP are fidelity consistent or constitute drift. The framework includes identification of who made the modification, at what level of delivery modifications are made, whether the modification was made to the content, context, or training and evaluation of the intervention, and the nature of the context or content modifications. Similar to the application of the SISTER compilation, these categories for treatment adaptation were embedded in the most structured tool and used to evaluate responses to the other two tools. Tool content and design were developed through an iterative process informed by research team meetings with implementation practitioners and the developer of the EBP. The piloted tools are available in Supplemental Files 2-4 and described below.

1. Brainstorming Log: This tool was the most open ended (Table 1). The Brainstorming Log was informed by a vocational education trainer's log (20) and literature on journaling as a qualitative data collection method (21). It consisted of six questions, one of which was multiple choice ("what is your role") and five free text. Before describing their activities, participants indicated the range of dates for which they reported. First, participants reported treatment adaptations made, describing content and context modifications in separate questions. Second, participants reported on barriers they encountered and strategies deployed (or proposed) to address those barriers.

2. Activity Log: This tool, based on Bunger et al. (8), was moderately structured and open ended (Table 1), using five same questions: participant role, date of the activity, time spent on the activity, the purpose, and the attendees. Unique to this tool, we asked about the intended outcome of the activity. This tool did not require participants to specify whether their activity was an implementation strategy or treatment adaptation.

3. Detailed Tracking Log: This tool was the most structured and detailed (Table 1). In addition to the questions from the Activity Log, participants were prompted to categorize each activity as an implementation strategy or treatment adaptation through pre-populated response options. Implementation strategies were organized into nine categories delineated by Waltz et al. (22) (see Supplemental File 3) and assigned a label from the SISTER compilation $(23,24)$. Participants reported on treatment adaptations according to Wiltsey Stirman and colleagues' (12)

\section{Measures}

Three measures of implementation outcomes, the Acceptability of Intervention Measure (AIM), Intervention Appropriateness Measure (IAM), and Feasibility of Intervention Measure (FIM) (25) were used 
to assess the likelihood that stakeholders might adopt these three tools. Each contain four items rated on a 5 -point scale ranging from $1=$ completely disagree to $5=$ completely agree. Summary scores for each measure were created by averaging responses, with higher values reflective of more favorable perceptions. AIM was used to measure the degree to which each tracking tool developed in this study was satisfactory to the stakeholders (current sample Cronbach's alpha of 0.97). IAM measured the relevance or perceived fit of each tracking tool (current sample Cronbach's alpha of 0.97). FIM measured the degree to which each tracking tool could be successfully utilized (current sample Cronbach's alpha of 0.96). These scales were followed by an open-ended question, "Please tell us why you rated this tracking method the way you did. What did you like/not like about it?"

The 6-item Adaptations to Evidence-Based Practices Scale (AES; (26)) was added to explore treatment adaptations using an established quantitative measure as a concurrent validity assessment with the tools in development. This measure includes six items rated on a 5 -point scale ranging from $0=$ not at all to $4=$ a very great extent. AES contains two subscales, "augmenting" adaptations ("I modify how I present or discuss components of the EBP") and "reducing/reordering" (I shorten/condense pacing of the EBP") adaptations. Cronbach's alpha for the total score was 0.94 , the augmenting subscale was 0.90 and the reducing/reordering subscale was 0.93 . Mean scores were calculated for each AES subscale, with higher scores indicating more adaptation.

\section{Pilot testing}

Setting and Participants. Our study capitalized on a pilot implementation of the Blues Program, an evidence-based cognitive behavioral group depression prevention program. This EBP is intended to promote engagement in pleasant activities and reduce negative cognitions among teens at risk of developing major depression $(27,28)$. The New York Foundling, a non-profit that offers services to children and families, includes an Implementation Support Center that provided oversight of the Blues Program implementation in New York state high schools. School-based mental health providers were trained by the Blues Program developer (PR) to facilitate group sessions. These three stakeholder groups, the Blues Program developer, implementation practitioners from New York Foundling, and mental health providers participated in our pilot by reporting on their Blues Program related activities via the three tracking tools. The Blues Program developer $(\mathrm{N}=1)$ was a $\mathrm{PhD}$ trained investigator with 30 years of posttraining experience. The implementation practitioners $(\mathrm{N}=3)$ all held master's degrees and had an average of 5.5 years of professional experience. The school-based mental health providers $(\mathrm{N}=7)$ also all had master's level training and worked in their profession an average of 4.3 years.

Data Collection. The tools were administered to the three participant groups across two cycles of the Blues Program each of which lasted six weeks. All groups were randomly assigned one tracking tool per week, distributed via an email that included a link to a web-based survey so that each tool was administered twice each cycle, in random order. Participants were instructed to complete the tracking tool by reflecting on the prior week's activities; they were given six days to record activities so as not to overlap 
with the next week's tool administration. At the end of data collection with each tool, participants completed the IAM, FIM, AIM, and AES. A \$10 per survey incentive was offered during the first round of data collection; this incentive was increased to $\$ 20$ for the second data collection cycle. Response rates for each round are reported in Table 2.

After data collection concluded, the first author conducted semi-structured interviews with participants that responded to the surveys: treatment developer $(\mathrm{N}=1)$, implementation practitioners from New York Foundling ( $\mathrm{N}=2)$, and school-based mental health providers $(\mathrm{N}=5)$. One implementation practitioner was on leave when interviews were conducted and two mental health providers left their positions prior to interviews and could not be contacted follow-up. The response rate among remaining participants was 100 percent for interviews ( $N=8)$. Interviews allowed for an in-depth exploration into stakeholders' experience with the tools. A semi-structured interview guide was prepared to capture information on: 1) perceived benefits of tracking, 2) tracking method preferences, 3) tracking process, 4) background or training for tracking, 5) tracking execution and completion, 6) general utility of tracking, and 7) contextual information (see Supplemental File 4 for full interview guide). Participants were emailed each tool so they could refer to them during the interview. Participants received a $\$ 40$ incentive upon interview completion. Interviews were recorded and transcribed.

\section{Data Analysis}

To assess participant perceptions of the tools, we compared scores on the AIM, IAM and FIM using a generalized estimating equation (GEE, a type of multilevel model). Analyses were completed the response level, rather than at the individual participant level. Scores on the measures were nested within weeks and roles and we examined fixed effects for role (treatment developer, implementation practitioners, and mental health providers) and tool (Detailed Tracking Log, Activity Log, and Brainstorming Log). As the measures did not show substantial skew or kurtosis, we used a linear model that assumes a normal distribution. We also used a similar analysis to see if the AES differed by role and tool. Because each of the predictors of interest had three categories, we ran each GEE twice, changing the reference group so we could examine all pairwise comparisons (i.e. Activity Log with Detailed Tracking Log, Detailed with Brainstorming Log, and Brainstorming Log with Activity Log). For the analyses, scores on the measures were only used if participants had reported at least $50 \%$ of the items. Three records were excluded because of missing data on the tools, while one record was excluded due to missing date information for a final sample of 59 responses.

We entered tool responses into an Access database for coding and created a codebook using established implementation frameworks and compilations. We categorized implementation strategies using the Waltz et al. (22) and SISTER compilations (23, 24); Proctor et al.'s (10) reporting recommendations for strategy specification; and the Wiltsey Stirman et al.'s (13) framework for treatment adaptations. Two research specialists (CWB, KM) trained by the study PI conducted dual independent coding and met weekly to resolve discrepancies. If consensus could not be reached among the two coders, the PI made a 
final decision. The resulting coded data offered a characterization of the reported activities based on alignment with implementation strategy reporting recommendations (10) and the treatment adaptation framework (12) (Table 3).

The first author and second author (LP), a sociologist with expertise in qualitative methods, conducted dual independent coding of all interview transcripts. A code book containing a priori codes based on the semi-structured interview guide was developed and iteratively refined with emergent codes added throughout the process. Code domains mapped onto the interview guide (Supplemental file 4). The coders held weekly consensus meetings to resolve discrepancies and reach agreement on additional emergent codes (29). Assigned codes were entered into ATLAS.ti (Version 7.1; (30)) and code reports were obtained and analyzed for main themes and illustrative quotes.

\section{Results}

\section{Acceptability, Appropriateness, and Feasibility}

Participants rated the three tracking methods as having moderate to good acceptability, appropriateness, and feasibility (Fig. 2). The Activity Log was significantly more acceptable ( $b=-.407, p=0.006)$ and feasible $(b=-.517, p<.001)$ but not more appropriate $(b=-.174, p=.394)$ than the Detailed Tracking Log. The Brainstorming Log did not significantly differ on acceptability, appropriateness and feasibility from the other two methods ( $p$ 's $>0.09$ ) except participants perceived the Activity Log as more feasible than the Brainstorming Log ( $b=-.346, p=0.015$; see Fig. 2$)$.

As shown in Fig. 2, mental health providers tended to report lower acceptability, appropriateness, and feasibility of all tracking methods compared to treatment developer and implementation practitioners (all p's $<0.05$ except the comparison of treatment developer with mental health providers on appropriateness, $p=0.122)$. However, the means on the AIM, IAM and FIM for mental health providers were between a 3 and 4 (on a 1-5 scale) indicating that they generally did not dislike the tracking methods but did not rate them as highly as the other participants.

\section{Adaptation to Evidence-Based Practice}

The extent to which participants reported making adaptations to the Blues Program in their role was low (Fig. 3). The nature of adaptations were more frequently augmenting factors rather than reducing/reordering. Responses to the AES in the Activity Log indicated the least amount of adaptation. This is consistent with the coding of the Activity Log data, which revealed only one treatment adaptation across 17 responses, compared to 4 in the Detailed Tracking Log and 20 in the Brainstorming Log (Table 3). As shown in Fig. 1, there were few significant differences between treatment adaptations reported by the participants except on the reordering subscale between the Activity Log and the Detailed Tracking Log. The treatment developer reported fewer treatment adaptations than the other two groups (all p's $<0.05$; Fig. 3 ) but the implementation practitioners and mental health providers did not significantly differ on reporting of treatment adaptations. 


\section{Qualitative findings}

\section{Tracking Process}

The average completion time across all three tracking methods was less than 15 minutes. The Activity Log had the longest completion time (mean $=14$ minutes), while the Detailed Tracking Log was the shortest (mean $=8.5$ minutes). The desire for brevity was expressed across all interview participants. One mental health provider noted that the ideal length of time to complete tracking is "ten minutes... in ten minutes you can do a lot of work." Another provider indicated "I have a lot of families I have to follow through with. The fact that it was not a long survey... was great."

All participants agreed the internet was the preferred mode of accessing the tools. One implementation practitioner recalled, "I thought that was super simple. I had a reminder in my calendar each week to do it." Although most participants indicated that tracking once a week was reasonable, there was some concern regarding frequency. The treatment developer reflected, "I don't know if it makes sense to wait until an entire Blues Program group is done. I don't think they would remember the changes that were made... [but] somehow weekly seems too much." Indeed, several mental health providers noted time and competing priorities as the largest barriers to tracking completion. One mental health provider reported,

We have children's plan progress notes, administrative things that we need to do, ...ongoing trainings, ...staff meetings, ...crises that we have to attend to that we can't account for... Those are probably some of the things that could prevent it from being completed on time. If I could make the decision when and how many times we get the survey... I would prefer it to be biweekly.

There was variation in how the tracking tools were interpreted by participants and the types of activities reported on each. One provider reflected, "when I would speak to my coworkers afterwards... everyone had different answers or different ways of viewing the questions... I just thought that was interesting how we all had very different perspectives, and different feedback to give in the actual surveys." An implementation practitioner indicated "with the Brainstorming Log... at times I connected it back a lot to the actual facilitation of the group... Because I wasn't facilitating at that time, I probably... gave a lot more information in the Detailed Tracking Logs." In general, mental health providers reported information on Blues Program sessions, whereas the treatment developer and implementation practitioners reported activities such as training, communication, preparation to implement the Blues Program at a new school, and other activities peripheral to EBP delivery.

\section{Brainstorming Log}

Participants indicated the Brainstorming Log was easy to use and provided an opportunity to reflect on recent activities. The treatment developer noted a preference for this tool, stating "...it's helpful when you're defining the content of the Blues and then the context and barriers is really clear, and the strategies, I like this one the best." Several participants reacted positively to the space to describe barriers and the open-ended questions. However, some mental health providers indicated a preference for multiple choice 
rather than open-ended questions; one suggested using multiple choice with an option to provide additional narrative. Several mental health providers felt there was a lack of clarity around the types of activities that should be reported, with one participant indicating they conducted some activities (e.g. providing "goodie bags" to students that completed the Blues Program), that were not captured by the questions available in this tracking tool.

\section{Activity Log}

Participants stated an overall strength of the Activity Log was its ease of use and indicated the questions in this tool provided enough structure and detail without being overly restrictive. An implementation practitioner indicated, "you're orienting it that way with purpose and outcome help to understand the intentionality behind what you're doing. So, I appreciated that about this one." Several participants noted the Activity Log was a helpful planning tool. A mental health provider reflected, "how much time you spent on it, that will allow me to plan ahead in regard to executing the program. Why did you choose this activity... what was the outcome... how would you know if it actually was effective... or did it do what it was supposed to do? That will be great information for me."

As with the Brainstorming Log, several participants perceived a lack of clarity in the Activity Log. The "date" prompt created confusion for several mental health providers, as they were uncertain if they should report calendar dates or Blues Program session number. One mental health provider noted, "...initially it seemed the easiest. But it actually is kind of confusing. I wasn't quite sure how to chunk up the actual problem or process I was going to write about."

\section{Detailed Tracking Log}

Several participants indicated the ability to select a pre-determined option was a strength of the Detailed Tracking Log. One mental health provider stated, "I liked that because then you were able to... narrow it down." The treatment developer indicated that the definitions of implementation strategies and treatment adaptations in this tool were helpful. The sentiment reflected by a mental health provider, "I don't know if it was just not relevant or if it was just the wording that didn't make it seem relevant" was expressed by several participants when describing the strategy labels. Another mental health provider noted the participant burden, "it's a lot of work...they're giving you options to help whoever is taking their survey compartmentalize what they did or how they adjusted it. Giving them...wording for it is helpful, or categories for it. But it is a lot of clicking around, and that might get confusing." The treatment developer indicated the difficulty in applying implementation science language, noting, "This is one where I needed to be educated... I was willing to try to read it and think about what it might have meant. But I did remember thinking... if the facilitators read this, they don't have the time... to try to understand."

\section{Recommendations for process improvement}

All participants indicated that additional background and training in the tracking process would have been useful. An implementation practitioner reflected, 
When I would send them [surveys] out, I did give a lot of rationale to the clinicians... Had I been able to look at them ahead of time, and really talk to you guys a little bit more about how they're going to be done, and questions that could come up by our frontline staff... to be able to better support them understanding the rationale, and what the differences are between implementation strategy and treatment adaptation. I know that they're not going to understand everything, nor am I. But I think had I had that opportunity it might have been a little bit easier for them [mental health providers].

Most participants indicated desire for the tracking process to be introduced during the Blues Program training. Several participants indicated there was a learning curve associated with tracking, as well as with distinguishing between implementation strategies and treatment adaptations. One implementation practitioner recalled, "in the beginning, I was still trying to figure that out. But I think over the past six months, I definitely see the distinction and I think if I were to have another opportunity doing this, I'd probably look at it a little differently."

There was variation not only in how the tracking tools were rated by the participants, but how tracking tools were interpreted. An implementation practitioner noted of the Brainstorming Log, "this kind of more free text boxes might have made more sense as like a post implementation strategy survey." Several participants indicated that they would be more likely to engage in tracking not only if they had a better understanding of the expectations, but also how tracking related to their work. One mental health provider noted, "I know it would be helpful for those coordinating the program, but if I knew it was for my benefit as well, it would motivate me more, definitely. To know I could reflect on it going into the next group I'm facilitating and seeing what adaptations I did or what may have worked or not." When asked how tracking could be more useful to their role, one mental health provider suggested, "it would probably be our supervisor to go through the feedback regarding the surveys."

\section{Discussion}

Given the importance of planned implementation strategy tailoring and treatment adaptation to promote the sustainability of EBPs [5], we developed and piloted three tools in a mixed-methods study designed to prospectively track implementation strategies and treatment adaptations. Participants across three groups perceived the tools to be acceptable, appropriate, and feasible, and offered useful feedback on formatting, content, administration process, and the ultimate purpose and function of the tools. This work builds on previous efforts to develop tracking tools and coding systems to capture detailed information about implementation strategy planning and tailoring efforts $(8,9)$.

Participants rated the Activity Log as the most feasible tool, and more acceptable than the Detailed Tracking Log. However, the Brainstorming Log revealed the most unique activities and barriers. Further, the Activity Log revealed only one treatment adaptation compared to 20 in the Brainstorming Log (Table 3), even though AES scores from the two tracking tools were similar (Fig. 3). This is surprising, as several mental health providers indicated they utilized the tracking tools to report on their Blues Program sessions. Even though the Activity Log was rated higher, it may not be the most effective tool for eliciting 
actionable data, especially treatment adaptations. This could be due to the lack of instruction to differentiate between implementation strategies and treatment adaptations as the other two tools had. Given the importance of tracking, further testing is needed to detect reporting differences when these instructions are present or absent. Given the potential for adaptations made by front line providers to improve the fit of the EBP to the needs of their local context $(14,31)$, having a tool that elicits detailed, reliable information about these adaptations is crucial for both researchers and practitioners.

It is important to note that all participants found this tracking to be quite different from anything they had previously done. The implementation practitioners gave the tracking tools the highest rating of the three stakeholder groups and had the most positive perceptions of tracking. Though all groups reported tracking had utility in their role, this was least salient among the mental health providers. Further, the providers most frequently perceived a lack of clarity when tracking their activities. Notably, this was the only stakeholder group not engaged in the tool development process. This perhaps points to the need to involve all stakeholder groups earlier in the process, and that tracking may be less meaningful if approached only as a data collection activity. Indeed, several providers noted they would be more likely to track their activities if they could connect tracking to service delivery. Several providers also indicated a desire to review their data and receive feedback from their practitioner. The needs of the stakeholders and the utility of tracking as an exercise, not only the data accumulated from tracking, are important considerations in designing the tools and their administration process.

The variability in how the tracking tools were interpreted and the types of activities reported on each may point to the need or desire for different versions of tracking tools at various stages of an implementation process. Most mental health providers treated tracking as an accompaniment to their session notes, and the content of their tracking tools focused on the delivery of the Blues Program content and the group sessions. This may suggest an important opportunity, especially given that several providers noted it was helpful to think prospectively about barriers and strategies to overcome them. Given the Brainstorming Log elicited the most barriers, it seems this tool would be best suited for this purpose. However, an implementation practitioner noted that this tool seemed like a post-implementation strategy survey. Further testing should reveal if different tracking tools elicit different kinds of data at various stages in the implementation process and if the nature of the role (e.g. delivering services vs. overseeing implementation) necessitates a unique kind of reporting. Understanding these nuances will better enable the field to design tools that allow for complete, detailed information about implementation strategies to be captured(10,32).

All participants noted that additional background and training about tracking would be helpful, and that there was a learning curve in the tracking process. Importantly, participants agreed that this type of training would be best delivered during training in the EBP, however one-time training would not be sufficient. Providers most frequently indicated a desire for follow-up, whether from implementation practitioners or clinical supervisors. Implementation practitioners, though engaged throughout the design and data collection process, also indicated desire to have additional opportunities to discuss tracking and 
receive more information. This illustrates that tracking is not a "set it and forget it" activity, rather is dynamic and requires more frequent follow-up and iteration.

While this pilot made strides towards developing tools to prospectively track treatment adaptations and implementation strategies in a low-burden manner, there were several limitations. First, due to the scale of the Blues Program implementation, our sample size in this pilot is small, with 11 participants across three roles. Additionally, the survey response rate varied week to week and across participant groups, ranging from $0-100 \%$. Participants were not consistently sent reminders to respond to the survey, so if they missed the email that week they may have been less likely to respond. New York Foundling appointed a program champion prior to the second round, which improved mental health provider engagement.

Finally, most participants were unfamiliar with implementation science terminology and identified needs for additional training, instructions, and examples to improve clarity. It also suggests a tension between the importance of reporting implementation strategies using consistent terminology and the need to find ways to track in a user-friendly way using terminology that might be clearer or more easily understood. Future work will serve to refine the tracking methods and test them with a larger group of stakeholders in various treatment contexts.

\section{Conclusions}

This study sought to fill methodological gaps that prevent stakeholders and researchers from discerning which activities are most important and when for promoting sustainment of EBPs. Tracking is essential for implementation research in order to understand how to build effective implementation strategies and to replicate previous effects. Tracking is also beneficial for implementation practice and clinical practice as it allows practitioners to better tailor efforts to their local context and to ensure EBP delivery is carried out with fidelity. Three tools show promise for these purposes, but additional work is needed to assess their psychometric and pragmatic properties and ensure they serve their intended purpose. Ultimately these tools could inform prospective tailoring of implementation strategies and treatment adaptations, which would promote EBP sustainment.

\section{Declarations}

\section{Ethics approval and consent to participate}

The Kaiser Permanente Washington Human Subjects Review Office determined this project was exempt from IRB review according to federal regulations.

\section{Consent for publication}

Not applicable. 
The datasets used in this study are available from the corresponding author upon reasonable request.

Competing interests

The authors declare they have no competing interests.

\section{Funding}

This study was funded by the Kaiser Permanente Washington Health Research Institute development funds.

\section{Authors' contributions}

CWB oversaw data collection, conducted participant interviews and data coding, and led the writing for this manuscript. LGP led the qualitative analysis work. SMWJ conducted the quantitative analysis. KDM conducted coding of survey data. BJP, SWS, and ARL served as consultants on this study, providing methodological and content expertise. PR contributed to the design of the tracking tools. CCL, the primary investigator, conceptualized and directed this project. All authors participated in conceptualizing, editing, and reviewing the manuscript.

Acknowledgements

We would like to acknowledge New York Foundling for their support of this study.

\section{Abbreviations}

AES - Adaptation of Evidence-Based Practices Scale

AIM - Acceptability of Intervention Measure

DSF - Dynamic Sustainability Framework

EBP - Evidence Based Practice

ERIC - Expert Recommendations for Implementing Change

FIM - Feasibility of Intervention Measure

IAM - Intervention Appropriateness Measure

PI - Primary Investigator

SISTER - School Implementation Strategies, Translating ERIC Resources

\section{References}


1. Baker R, Camosso-Stefinovic J, Gillies C, Shaw EJ, Cheater F, Flottorp S, et al. Tailored interventions to address determinants of practice. Cochrane Database Syst Rev. 2015;4.

2. Powell BJ, Beidas RS, Lewis CC, Aarons GA, McMillen JC, Proctor EK, et al. Methods to improve the selection and tailoring of implementation strategies. J Behav Health Serv Res. 2017;44(2):177-94.

3. Lewis CC, Scott K, Marti CN, Marriott BR, Kroenke K, Putz JW, et al. Implementing MeasurementBased Care (iMBC) for depression in community mental health: a dynamic cluster randomized trial study protocol. Implement Sci. 2015;10:127.

4. Kilbourne AM, Almirall D, Eisenberg D, Waxmonsky J, Goodrich DE, Fortney JC, et al. Protocol: Adaptive Implementation of Effective Programs Trial (ADEPT): cluster randomized SMART trial comparing a standard versus enhanced implementation strategy to improve outcomes of a mood disorders program. Implement Sci. 2014;9(1):132.

5. Chambers DA, Glasgow RE, Stange KC. The dynamic sustainability framework: addressing the paradox of sustainment amid ongoing change. Implement Sci. 2013;8:117.

6. Wiltsey Stirman S, Finley EP, Shields N, Cook J, Haine-Schlagel R, Burgess JF, Jr., et al. Improving and sustaining delivery of CPT for PTSD in mental health systems: a cluster randomized trial. Implement Sci. 2017;12(1):32.

7. Wensing M. The Tailored Implementation in Chronic Diseases (TICD) project: introduction and main findings. Implement Sci. 2017;12(1):5.

8. Bunger AC, Powell BJ, Robertson HA, MacDowell H, Birken SA, Shea C. Tracking implementation strategies: a description of a practical approach and early findings. Health Res Policy Syst. 2017;15(1):15.

9. Boyd MR, Powell BJ, Endicott D, Lewis CC. A method for tracking implementation strategies: an exemplar implementing measurement-based care in community behavioral health clinics. Behav Ther. 2017;(advance online publication)(4):525-37.

10. Proctor EK, Powell BJ, McMillen JC. Implementation strategies: recommendations for specifying and reporting. Implement Sci. 2013;8:139.

11. Powell BJ, Waltz TJ, Chinman MJ, Damschroder LJ, Smith JL, Matthieu MM, et al. A refined compilation of implementation strategies: results from the Expert Recommendations for Implementing Change (ERIC) project. Implement Sci. 2015;10:21.

12. Wiltsey Stirman S, Miller CJ, Toder K, Calloway A. Development of a framework and coding system for modifications and adaptations of evidence-based interventions. Implement Sci. 2013;8(1):65.

13. Wiltsey Stirman S, Baumann AA, Miller CJ. The FRAME: an expanded framework for reporting adaptations and modifications to evidence-based interventions. Implement Sci. 2019;14(1):58.

14. Chambers D, Norton WE. The Adaptome: Advancing the Science of Intervention Adaptation. Am J Prev Med. 2016;51(4 (Suppl 2)):s124-s31.

15. Edwards P. Questionnaires in clinical trials: guidelines for optimal design and administration. Trials. 2010;11(1):2. 
16. Demetriou C, Ozer BU, Essau CA. Self-report questionnaires. 2015. In: The encyclopedia of clinical psychology [Internet]. John Wiley \& Sons, Inc.; [1-6].

17. Reja U, Manfreda KL, Hlebec V, Vehovar V. Open-ended vs. close-ended questions in web questionnaires. Dev Appl Stat. 2003;19(1):106-17.

18. Cook CR, Lyon AR, Locke J, Waltz T, Powell BJ. Adapting a compilation of implementation strategies to advance school-based implementation research and practice. Prev Sci. 2019.

19. Lyon AR, Cook CR, Locke J, Davis C, Powell BJ, Waltz TJ. Importance and feasibility of an adapted set of strategies for implementing evidence-based mental health practices in schools. Forthcoming.

20. Albright L, R. CB. Formative evaluation of a training curriculum for vocational education and special services personnel. Annual Meeting of the American Educational Research Association; New Orleans, LA: U.S. Department of Education; 1988.

21. Tuckett AG, Stewart DE. Collecting qualitative data: part II journal as a method: experience, rationale and limitations. Contemp Nurse. 2004;16(3):240-51.

22. Waltz TJ, Powell BJ, Matthieu MM, Damschroder LJ, Chinman MJ, Smith JL, et al. Use of concept mapping to characterize relationships among implementation strategies and assess their feasibility and importance: results from the Expert Recommendations for Implementing Change (ERIC) study. Implement Sci. 2015;10:109.

23. Cook CR, Lyon AR, Locke J, Waltz TJ, Powell BJ. Adapting a compilation of implementation strategies to advance school-based implementation research and practice. Prevention Science. in press.

24. Lyon AR, Cook CR, Locke J, Davis C, Powell BJ, Waltz TJ. Importance and feasibility of an adapted set of strategies for implementing evidence-based mental health practices in schools. under review.

25. Weiner BJ, Lewis CC, Stanick C, Powell BJ, Dorsey CN, Clary AS, et al. Psychometric assessment of three newly developed implementation outcome measures. Implement Sci. 2017;12(1):108.

26. Lau A, Barnett M, Stadnick N, Saifan D, Regan J, Wiltsey Stirman S, et al. Therapist report of adaptations to delivery of evidence-based practices within a system-driven reform of publicly funded children's mental health services. J Consult Clin Psychol. 2017;85(7):664-75.

27. Stice E, Rohde P, Gau JM, Wade E. Efficacy trial of a brief cognitive-behavioral depression prevention program for high-risk adolescents: effects at 1- and 2-year follow-up. J Consult Clin Psychol. 2010;78(6):856-67.

28. Stice E, Rohde P, Seeley JR, Gau JM. Brief cognitive-behavioral depression prevention program for high-risk adolescents outperforms two alternative interventions: a randomized efficacy trial. J Consult Clin Psychol. 2008;76(4):595-606.

29. Hill CE, Knox S, Thompson BJ, Williams EN, Hess SA, Ladany N. Consensual qualitative research: an update. J Couns Psychol. 2005;52(2):196-205.

30. Ringmayr TG. ATLAS.ti version 7.1.0. Berlin, Germany: ATLAS.ti Scientific Software Development $\mathrm{GmbH} ; 2013$. 
31. Lau AS. Making the case for selective and directed cultural adaptations of evidence-based treatments: Examples from parent training. Clin Psychol-Sci Pr. 2006;13.

32. Powell BJ, Fernandez ME, Williams NJ, Aarons GA, Beidas RS, Lewis CC, et al. Enhancing the Impact of Implementation Strategies in Healthcare: A Research Agenda. Front Public Health. 2019;7:3.

\section{Tables}

Table 1: Structure of self-report measures and reporting recommendations by tracking tool

\begin{tabular}{|c|c|c|c|c|c|c|c|}
\hline & & \multicolumn{6}{|c|}{\begin{tabular}{|l|} 
Not Structured \\
Structured
\end{tabular}} \\
\hline & & $\begin{array}{c}\text { No } \\
\text { Questions }\end{array}$ & Brainstorming & $\begin{array}{l}\text { Unstructured } \\
\text { Interview }\end{array}$ & $\begin{array}{l}\text { Structured } \\
\text { Interview }\end{array}$ & $\begin{array}{c}\text { Self- } \\
\text { Report } \\
\text { (Open } \\
\text { Ended) }\end{array}$ & \begin{tabular}{|c} 
Self-Report \\
(Close- \\
Ended)
\end{tabular} \\
\hline \multirow{11}{*}{$\begin{array}{l}\text { Brainstorming } \\
\text { Log }\end{array}$} & Name & $\mathrm{x}$ & & & & & \\
\hline & Define & $\mathrm{x}$ & & & & & \\
\hline & Actor & & $\mathrm{x}$ & & & & \\
\hline & Action & & $\mathrm{x}$ & & & & \\
\hline & Target & $\mathrm{x}$ & & & & & \\
\hline & Dose & $\mathrm{x}$ & & & & & \\
\hline & Temporality & & $\mathrm{x}$ & & & & \\
\hline & Outcome & $\mathrm{x}$ & & & & & \\
\hline & Justification & $\mathrm{x}$ & & & & & \\
\hline & Barriers & & $\mathrm{x}$ & & & & \\
\hline & Adaptation & & $\mathrm{X}$ & & & & \\
\hline \multirow[t]{11}{*}{ Activity Log } & Name & $\mathrm{x}$ & & & & & \\
\hline & Define & $\mathrm{x}$ & & & & & \\
\hline & Actor & & & & & $\mathrm{x}$ & \\
\hline & Action & & & & & $\mathrm{x}$ & \\
\hline & Target & $\mathrm{x}$ & & & & & \\
\hline & Dose & & & & & $\mathrm{x}$ & \\
\hline & Temporality & & & & & $\mathrm{x}$ & \\
\hline & Outcome & & & & & $\mathrm{X}$ & \\
\hline & Justification & $\mathrm{x}$ & & & & & \\
\hline & Barriers & & & & & $\mathrm{x}$ & \\
\hline & Adaptation & $\mathrm{x}$ & & & & & \\
\hline \multirow{11}{*}{$\begin{array}{c}\text { Detailed } \\
\text { Tracking Log }\end{array}$} & Name & & & & & & Standardized \\
\hline & Define & $\mathrm{x}$ & & & & & \\
\hline & Actor & & & & & & $\mathrm{x}$ \\
\hline & Action & & & & & & $\mathrm{X}$ \\
\hline & Target & $\mathrm{x}$ & & & & & \\
\hline & Dose & & & & & & $\mathrm{x}$ \\
\hline & Temporality & & & & & & $\mathrm{x}$ \\
\hline & Outcome & & & & & & $\mathrm{x}$ \\
\hline & Justification & $\mathrm{x}$ & & & & & \\
\hline & Barriers & & & & & & $\mathrm{x}$ \\
\hline & Adaptation & & & & & & Standardized \\
\hline
\end{tabular}


Table 2: Survey Response Rates

\begin{tabular}{|l|c|c|c|c|c|c|}
\hline & \multicolumn{3}{|c|}{ Round 1 } & \multicolumn{3}{c|}{ Round 2 } \\
\hline & $\begin{array}{c}\text { Activity } \\
\text { Log }\end{array}$ & $\begin{array}{c}\text { Brainstorming } \\
\text { Log }\end{array}$ & $\begin{array}{c}\text { Detailed } \\
\text { Tracking } \\
\text { Log }\end{array}$ & $\begin{array}{c}\text { Activity } \\
\text { Log }\end{array}$ & $\begin{array}{c}\text { Brainstorming } \\
\text { Log }\end{array}$ & $\begin{array}{c}\text { Detailed } \\
\text { Tracking } \\
\text { Log }\end{array}$ \\
\hline $\begin{array}{l}\text { Mental Health } \\
\text { Provider }\end{array}$ & $44.4 \%$ & $33.3 \%$ & $66.7 \%$ & $100 \%$ & $100 \%$ & $100 \%$ \\
\hline $\begin{array}{l}\text { Implementation } \\
\text { Practitioner }\end{array}$ & $83.3 \%$ & $16.7 \%$ & $66.7 \%$ & $50 \%$ & $83.3 \%$ & $83.3 \%$ \\
\hline $\begin{array}{l}\text { Treatment } \\
\text { Developer }\end{array}$ & $100 \%$ & $66.6 \%$ & $100 \%$ & $0 \%$ & $0 \%$ & $0 \%$ \\
\hline
\end{tabular}

Table 3: Barriers and activities reported by Tracking Tool

\begin{tabular}{|c|c|c|c|}
\hline Role & Barriers & $\begin{array}{l}\text { Implementation } \\
\text { Strategies }\end{array}$ & $\begin{array}{c}\text { Treatment } \\
\text { Adaptations }\end{array}$ \\
\hline \multicolumn{4}{|c|}{ Brainstorming Log $(\mathrm{N}=24)$} \\
\hline $\begin{array}{l}\text { Mental Health Provider } \\
\text { Implementation }\end{array}$ & 7 & 1 & 8 \\
\hline Practitioner & 26 & 22 & 9 \\
\hline Treatment Developer & 2 & 1 & 3 \\
\hline \multicolumn{4}{|c|}{ Activity $\log (\mathrm{N}=17)$} \\
\hline $\begin{array}{l}\text { Mental Health Provider } \\
\text { Implementation }\end{array}$ & 3 & 2 & 1 \\
\hline Practitioner & 6 & 9 & 0 \\
\hline Treatment Developer & 0 & 1 & 0 \\
\hline Total & 9 & 13 & 1 \\
\hline \multicolumn{4}{|c|}{ Detailed Tracking Log $(\mathrm{N}=22)$} \\
\hline $\begin{array}{l}\text { Mental Health Provider } \\
\text { Implementation }\end{array}$ & 1 & 7 & 3 \\
\hline Practitioner & 0 & 9 & 1 \\
\hline Treatment Developer & 1 & 3 & 0 \\
\hline Total & 2 & 19 & 4 \\
\hline
\end{tabular}

Note: $\mathrm{N}$ represents number of responses per log. Each response may report more than one type of activity.

\section{Figures}




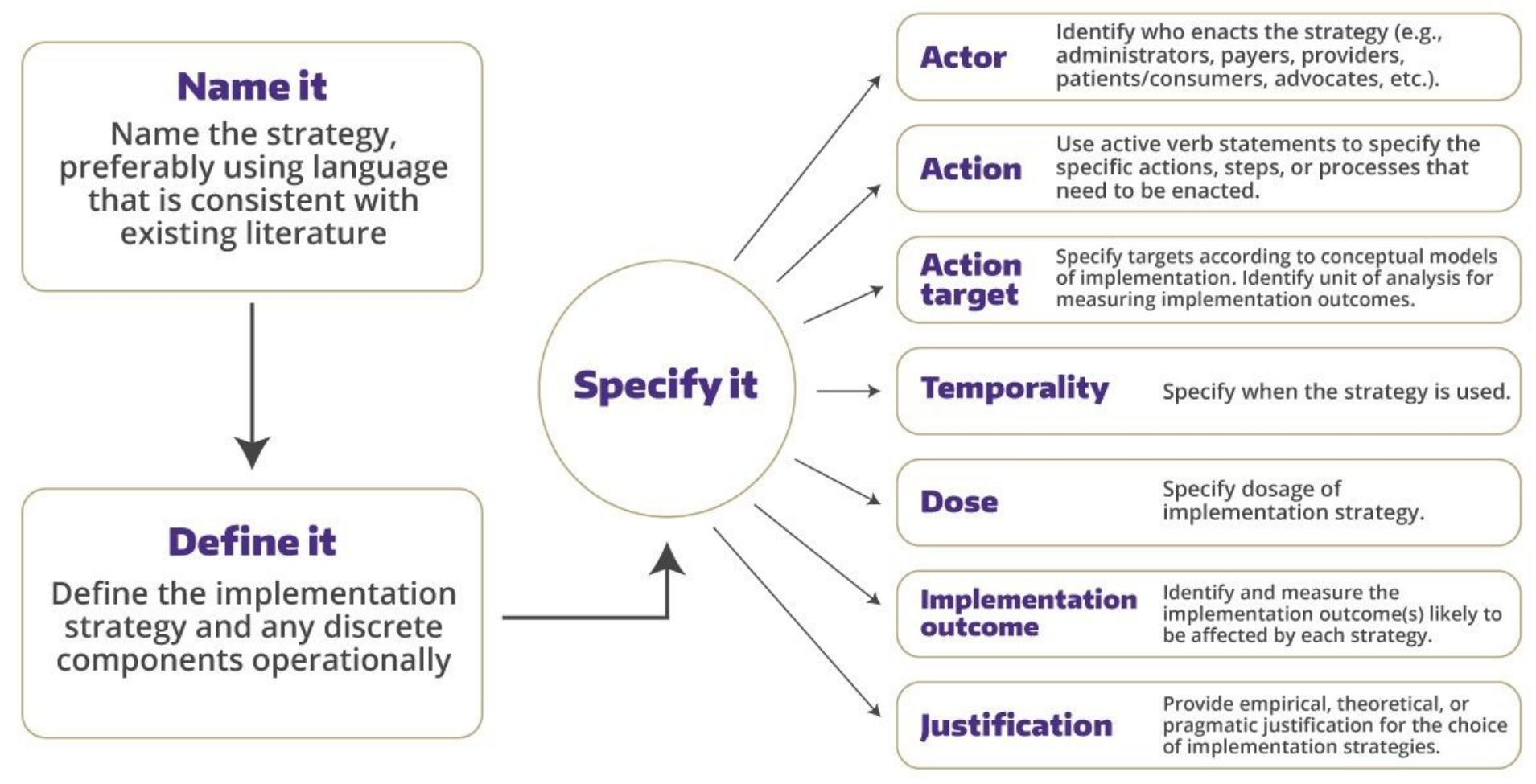

\section{Figure 1}

Implementation strategy reporting recommendations Note: Adapted from Proctor EK, Powell BJ, McMillen JC. Implementation strategies: Recommendations for specifying and reporting. Implement Sci. 2013;8(139). Reproduced with Permission from Bryan Weiner, PhD. 

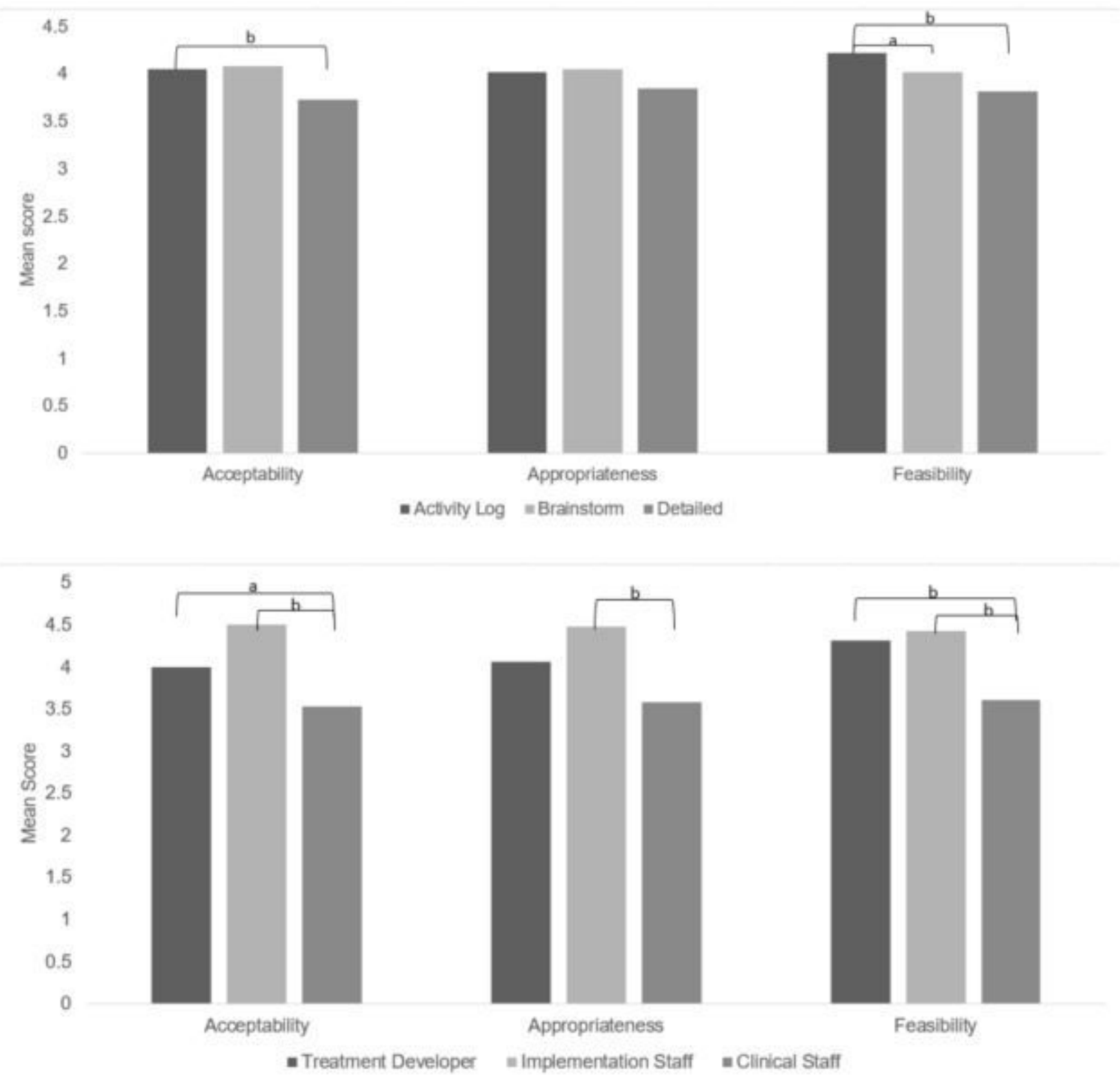

Figure 2

Means for Acceptability of Intervention Measure, Intervention Appropriateness Measure, and Feasibility of Intervention Measure by tracking method. a=pairwise comparison was significant at the $p<0.05$ level. $b=$ pairwise comparison was significant at the $p<0.01$ level 

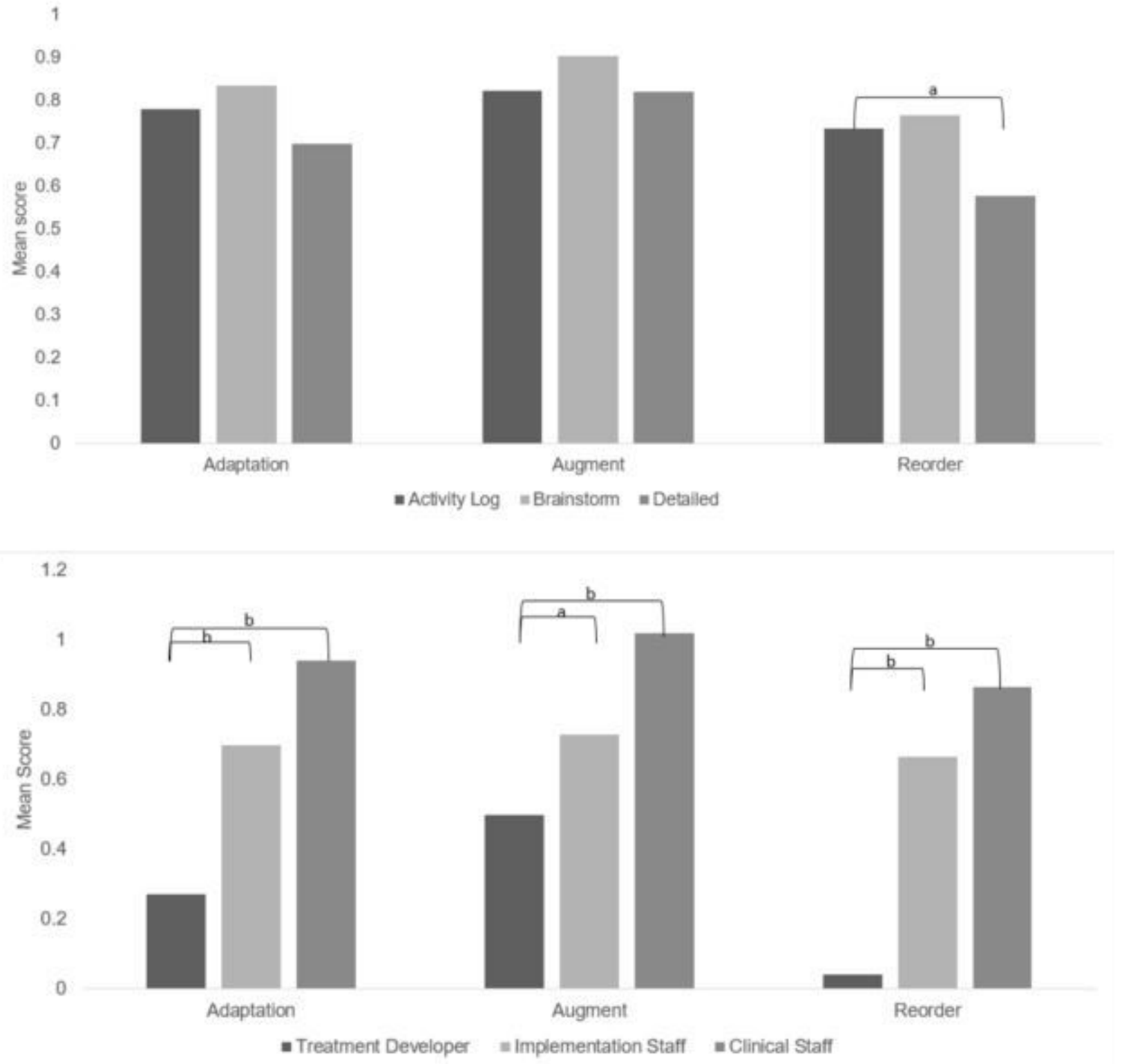

\section{Figure 3}

Means for the Adaptation of Evidence Based Practice Scale. a=pairwise comparison was significant at the $p<0.05$ level. $b=$ pairwise comparison was significant at the $p<0.01$ level

\section{Supplementary Files}

This is a list of supplementary files associated with this preprint. Click to download.

- SupplementalFile1CodeList.docx

- SupplementalFile3ActivityLog.docx

- SupplementalFile4DetailedTrackingLog.docx

- SupplementalFile5InterviewGuide.docx 
- SupplementalFile6StaRIchecklistforauthorcompletion.docx

- SupplementalFile2BrainstormingLog.docx 